\title{
MONITORAMENTO DE APLICAÇÕES NO AMBIENTE DE AUTOMAÇÃO INDUSTRIAL *
}

Anderson Ayres Bittencourt ${ }^{1}$ Claudio Antunes de Oliveira²

\section{Resumo}

O monitoramento de sistemas e ativos de automação sempre existiu de forma rudimentar e descentralizada. A análise desses dados tanto quanto sua obtenção é um desafio para as empresas atuais. Foi a partir deste princípio que nosso projeto foi desenvolvido.

Este trabalho tem como objetivo demonstrar a solução de Monitoramento online e preditivo implementada na ArcelorMittal Aços Planos da América do Sul, onde desde o início de sua implementação pudemos capturar benefícios operacionais, melhoria dos processos, otimização, eliminação e/ou diminuição de indisponibilidade de plantas, entre diversos outros retornos que serão apresentados.

Palavras-chave: Monitoramento online; Histórico de Monitoramento; Predição; Análise de Dados; IloT; Alarmes; Automatização de Ações.

\section{APPLICATION MONITORING IN THE INDUSTRIAL AUTOMATION ENVIRONMENT}

\section{Abstract}

The monitoring of automation systems and assets has always existed in a rudimentary and decentralized way. And analyzing such data as much as getting it is a challenge for today's businesses. It was from this principle that our project was developed.

This work aims to demonstrate the online and predictive monitoring solution implemented at ArcelorMittal Flat Carbon South America, where from the beginning of its implementation we were able to capture operational benefits, process improvement, optimization, elimination and / or reduction of plant unavailability among several others returns that will be presented.

Keywords: Online monitoring; Monitoring History; Prediction; Data analysis; IloT; Alarms; Automated Actions.

1 Graduado em Redes de Computadores e Pós-graduado em Gerenciamento de TI, Especialista na área de Engenharia de Automação de Processos, ArcelorMittal Tubarão, Vitória, Espírito Santo, Brasil.

2 Graduado em Engenharia Elétrica, Especialista na área de Engenharia de Automação de Processos, ArcelorMittal Tubarão, Vitória, Espírito Santo, Brasil. 


\section{INTRODUÇÃO}

O monitoramento completo de um ambiente seja em TI, seja em TA, sempre foi um desafio para os especialistas devido a sua enorme quantidade de dispositivos e aplicativos, sua grande heterogeneidade, a quantidade de informações geradas e principalmente como essa informação seria analisada e utilizada.

Isso fica ainda mais difícil no ambiente de TA, pois se tratam de redes e equipamentos legados, com protocolos pouco comerciais e dispositivos que por muitas vezes não possuem interfaces de monitoramento.

E cada dia fica mais claro para as indústrias que monitoramento é diretamente ligado a disponibilidade, otimização e retorno financeiro.

Foi desta demanda crescente que nasceu o projeto do MON-X (Monitoramento de Sistemas de Automação da ArcelorMittal). Um pacote de soluções desenvolvida pela ArcelorMittal para a obtenção e tratamento dos dados da planta industrial e que utiliza produtos opensource para a parte de gestão, interface web e banco de dados.

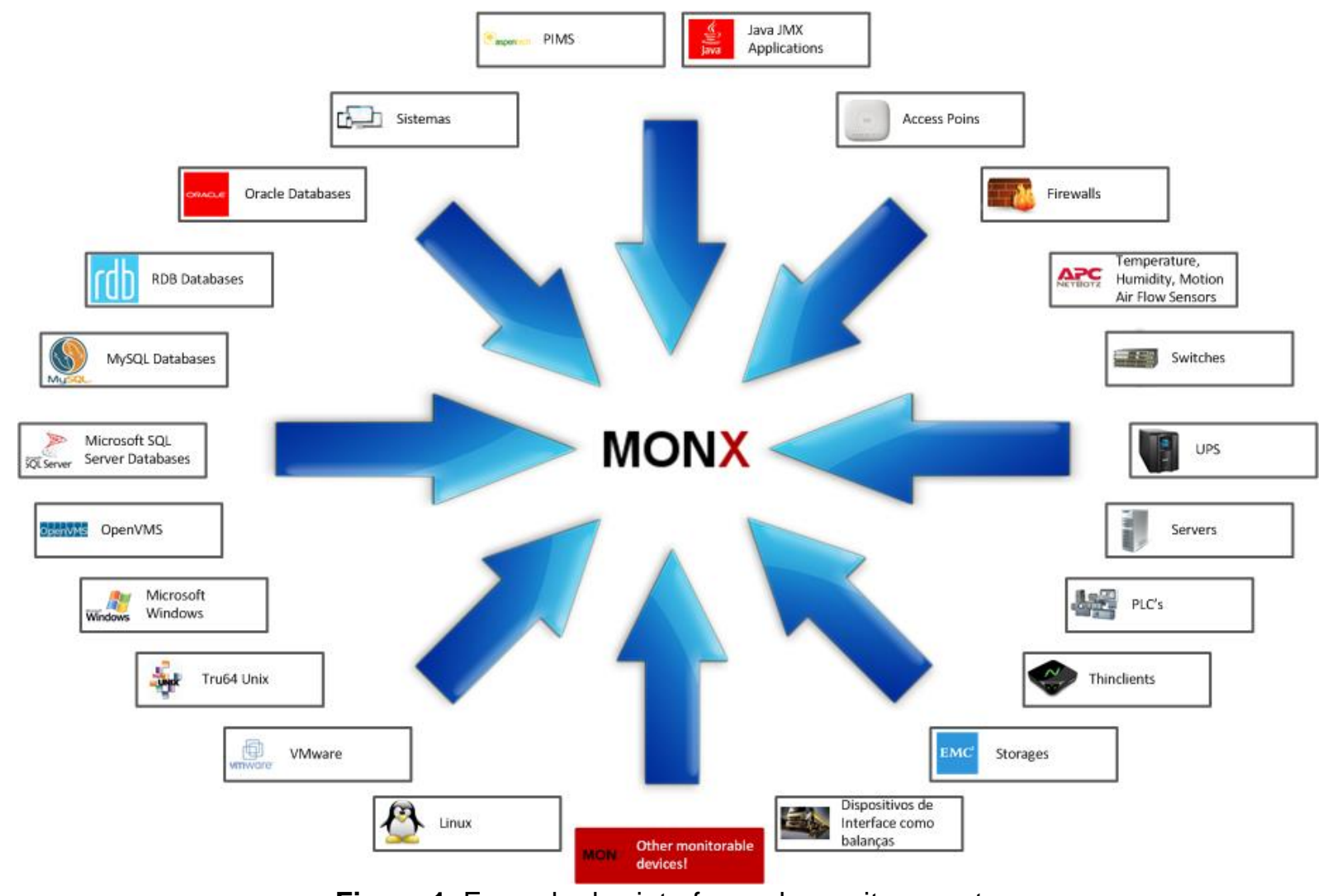

Figura 1. Exemplo das interfaces de monitoramento

\section{MATERIAIS E MÉTODOS}

Com o objetivo de monitorar de forma centralizada os dados das plantas da ArcelorMittal, foi selecionada uma suíte de produtos opensource, entre eles o Zabbix $^{1}$ e o MySQL, para concentrar nossos dados e a partir daí fazer a geração de KPI's e alarmes.

Com as ferramentas de gestão selecionadas, começaram os desenvolvimentos para extração de dados das mais diversas fontes incluindo dispositivos loT/IIoT, conforme pode ser observado na Figura 2. 


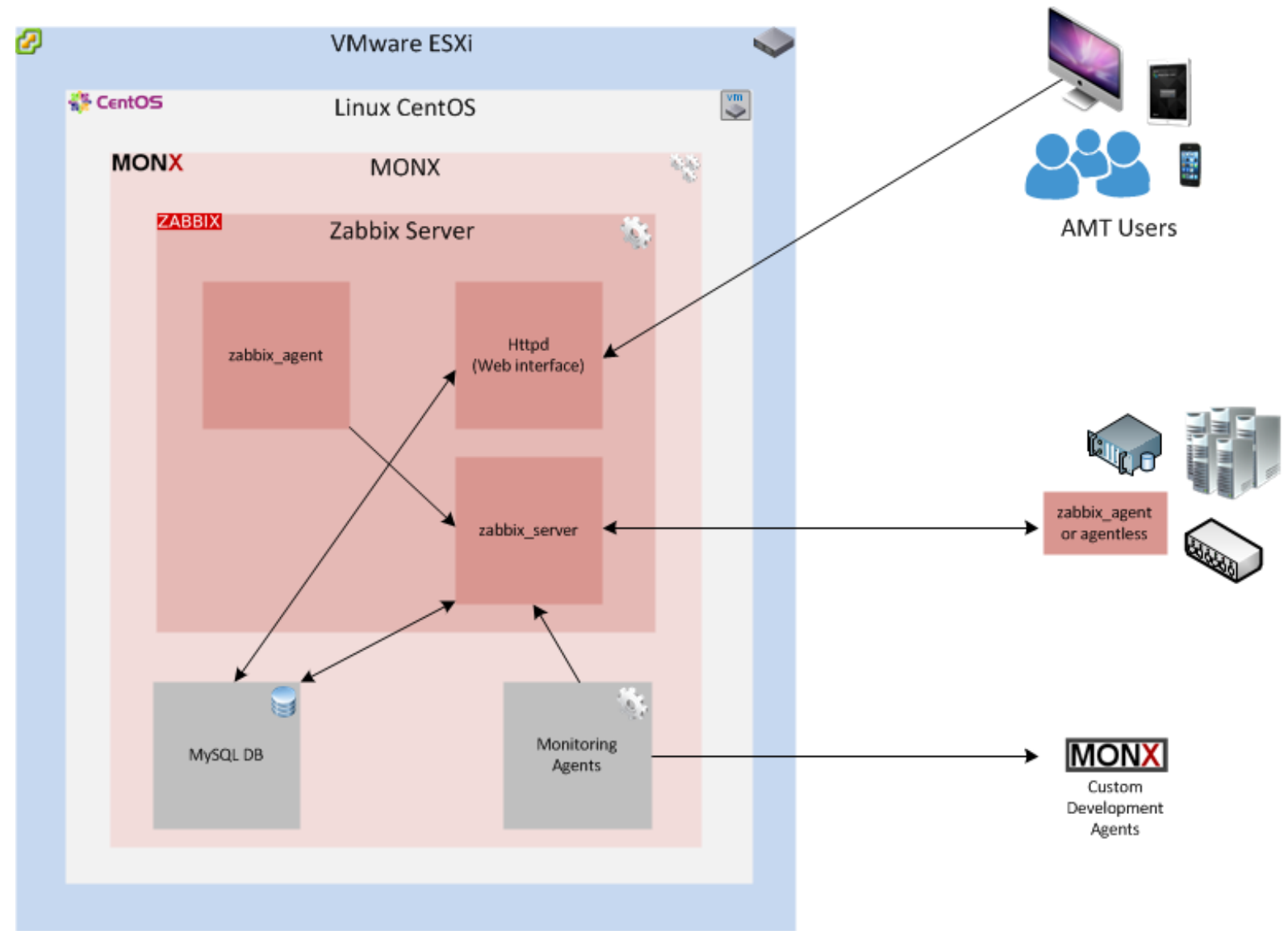

Figura 2. Arquitetura da Solução

\subsection{Redes Profibus}

A solução que encontrada para monitoramento de redes Profibus online foi utilizar dispositivos Profilink que ficam anexados a rede de forma ininterrupta e fornecem informações através do protocolo SNMP.

Anteriormente, devido ao alto custo e a pouca oferta de equipamentos inteligentes para redes Profibus, o monitoramento de redes Profibus era feito através de maletas portáteis e os dados coletados eram apenas do período em que a maleta era conectada a rede.

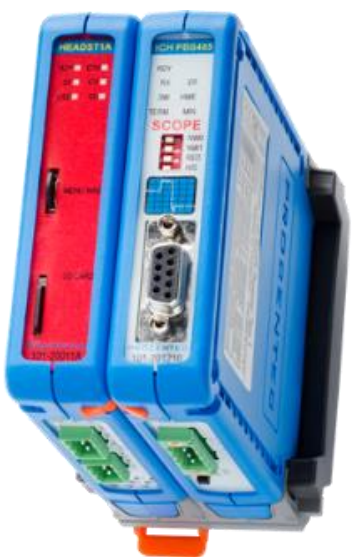

Figura 3. Dispositivo utilizado para monitoramento de Redes Profibus (Profilink) 


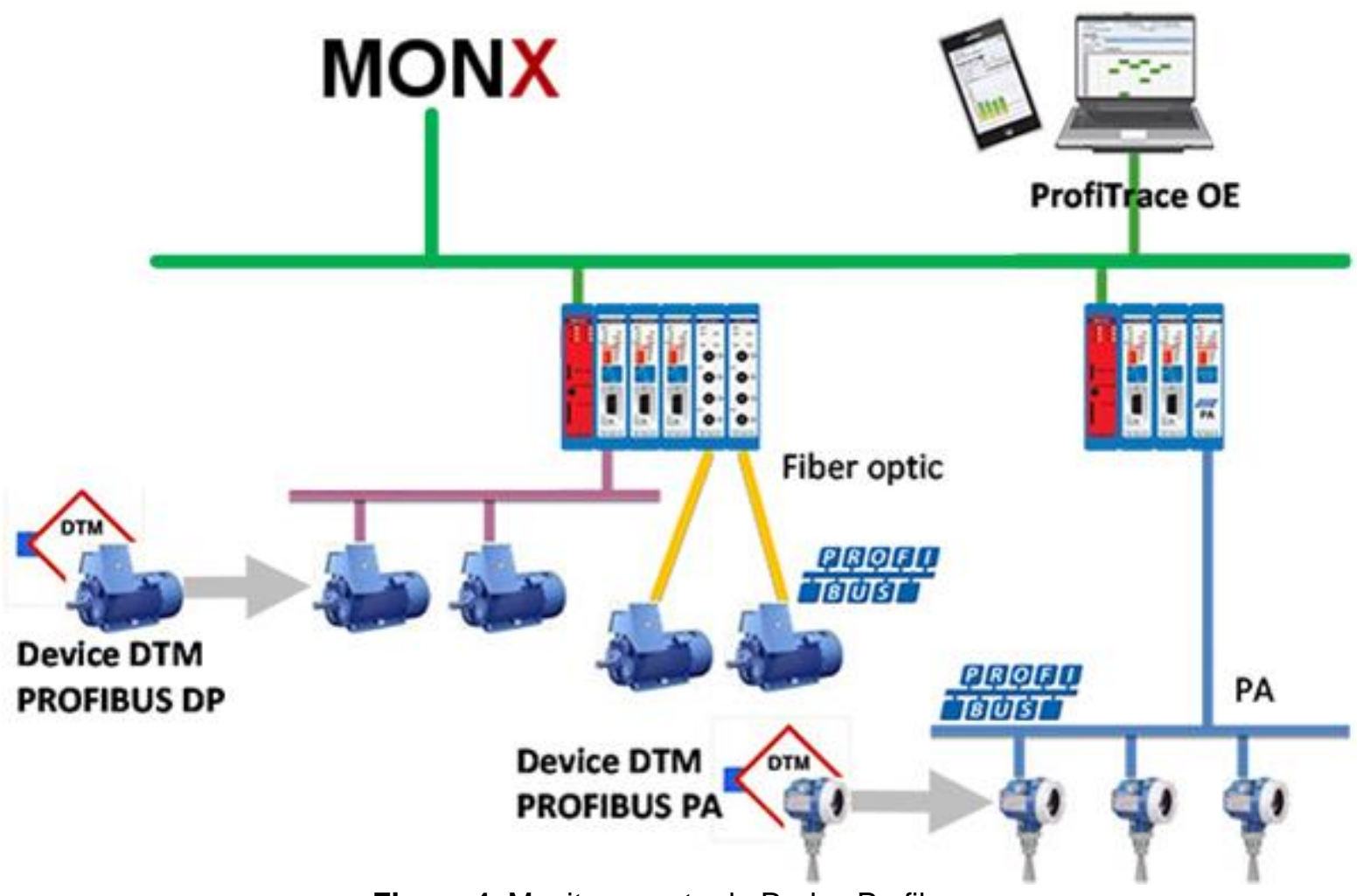

Figura 4. Monitoramento de Redes Profibus

\subsection{Dispositivos loT}

Dispositivos IoT e IloT são utilizados na plataforma através de desenvolvimento próprio, onde utilizando a API de comunicação do Zabbix, foi desenvolvida toda parte de coleta e tratamento de dados baseado em $\mathrm{c}, \mathrm{C}++$, python entre outras linguagens e a partir daí foram enviados os dados para a plataforma do MON-X; incluindo sensores de todos os tipos que são plugados a esses dispositivos e grande parte do desenvolvimento está sendo baseado no Raspberry PI, conforme pode ser observado na Figura 5. 


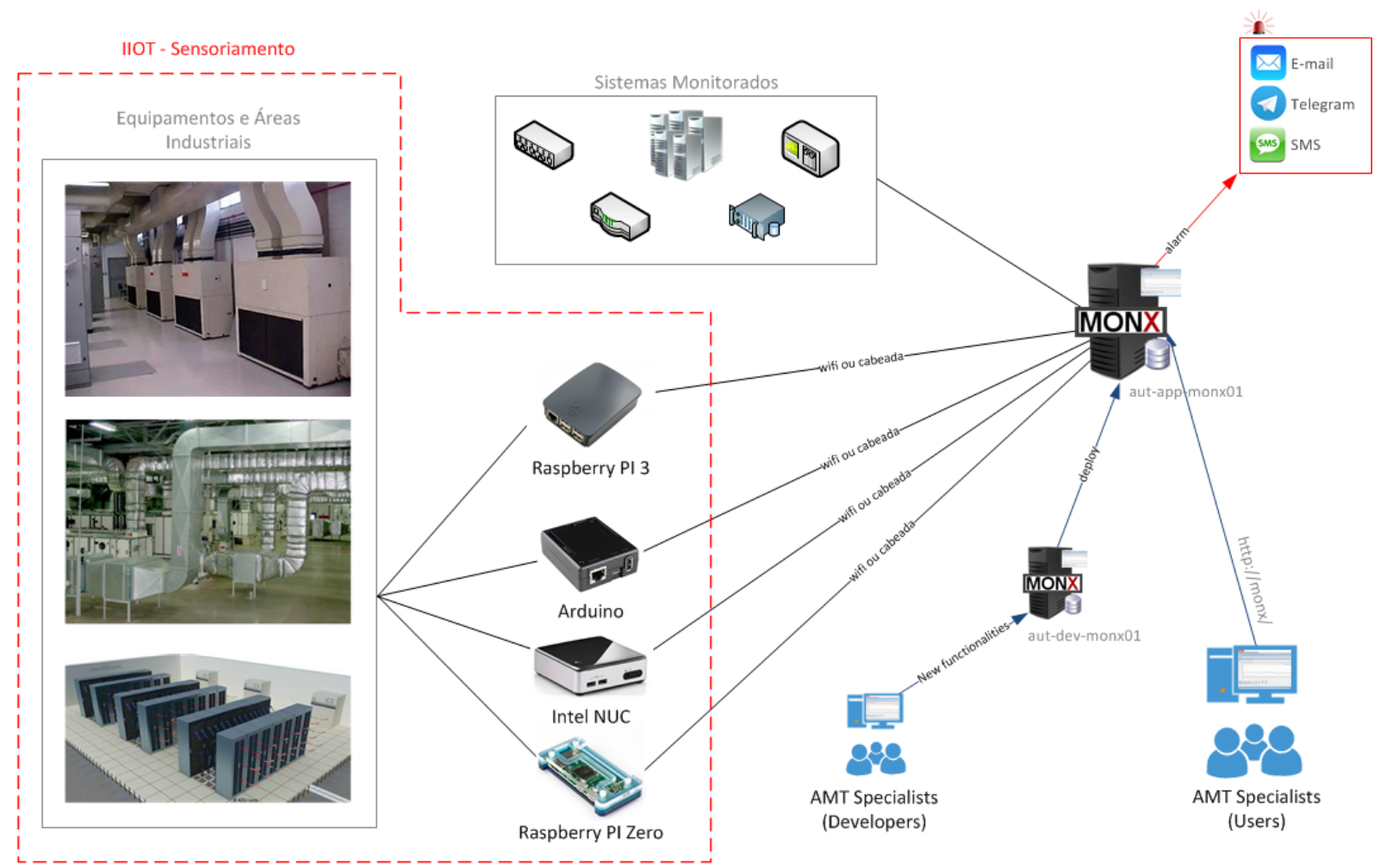

Figura 5. Monitoramento com dispositivos IoT

\subsection{Dispositivos Full Gauge}

Para dispositivos Full Gauge, foi desenvolvido um driver de comunicação que lê diretamente do SITRAD (Software de Monitoramento/Configuração da Full Gauge) e encaminha os dados para o ambiente de monitoramento.

Toda integração através do driver MON-X é feita em .NET, inclusive o pooling das coletas e controle de threads. O Zabbix recebe o dado tratado e pronto para ser utilizado.

\subsection{Sistemas Legados}

Todo ambiente foi planejado para atender a ambientes legados.

Este requisito era premissa inicial do projeto, a fim de substituir o sistema de monitoramento anterior (SGHOST) e prover uma plataforma atualizada e com mais funcionalidades.

Com a arquitetura apresentada, foi possível cobrir todo o parque de legados, chegando a $100 \%$ dos sistemas OpenVMS ${ }^{2}$ e Tru6 $4^{3}$ monitorados, inclusive com monitoramento dos sensores físicos de máquinas Alpha Server através de agentes desenvolvidos pela ArcelorMittal para este fim.

Agora os sistemas legados contam com a mesma atenção dos sistemas novos, monitoramento contínuo, prevenção de falhas e alarmes customizados.

\subsection{Integração de Sites remotos}

Uma questão crítica para o projeto era "Como consolidar os dados de todas as plantas e ter um cockpit único de eventos e ações?".

Novamente o planejamento foi colocado a prova e hoje 3 sites trabalham $100 \%$ integrados: Tubarão, Vega do Sul e Contagem. 
Os dados são analisados em tempo real e cockpits contendo todos os sites são providos a qualquer equipe a qualquer hora.

Inclusive o troubleshoot de problemas pode ser feito por equipes remotas e com apoio imediato pelo simples fato dos dados já estarem na mão deles e acessíveis de qualquer um dos sites.

Troubleshoots passaram de horas ou até mesmo dias, para minutos. E a mesma informação que um expert no assunto visualiza é exibida de forma simplificada para o analista da área, possibilitando a ambos falarem a mesma linguagem.

Abaixo temos uma representação de como funciona a integração Tubarão x VEGA:

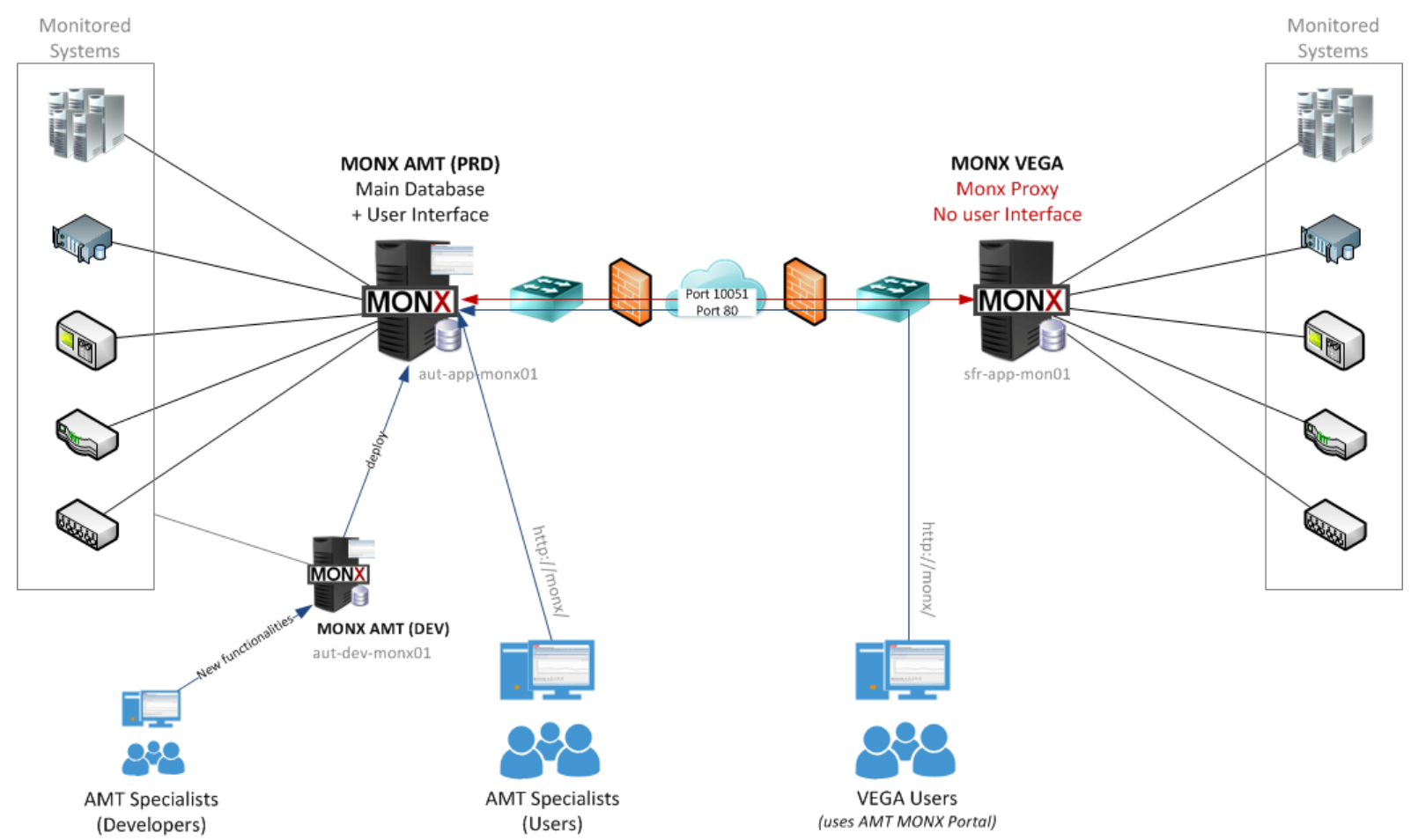

Figura 6. Integração entre os sites

\subsection{Segurança}

O item segurança é tratado com bastante seriedade pela ferramenta escolhida.

Os itens relevantes sobre a arquitetura são:

- Controle de Acesso baseado no Active Directory.

- Permissão customizada para cada objeto dentro do sistema, permitindo o controle granular de permissões de acesso.

- Transmissão de dados criptografada entre os agentes e o servidor.

- Portas padronizadas e não ranges, permitindo um ajuste correto entre os firewalls.

- Necessidade de liberação de apenas 2 portas entre sites remotos para sincronização dos dados dos sites.

- Áreas com demanda de isolamento atendidas por proxy. Melhor controle de segurança e limitação de apenas 1 ponto de acesso ao servidor principal. (Apenas o proxy se comunica com o servidor, as estações todas se comunicam com o proxy) 


\subsection{Gestão à Vista (Cockpit de Monitoramento)}

\subsection{1 - TV's Android nas salas}

Foram implantadas TV's em todas as salas da automação, com telas específicas para cada área de interesse e contendo informações online da planta:

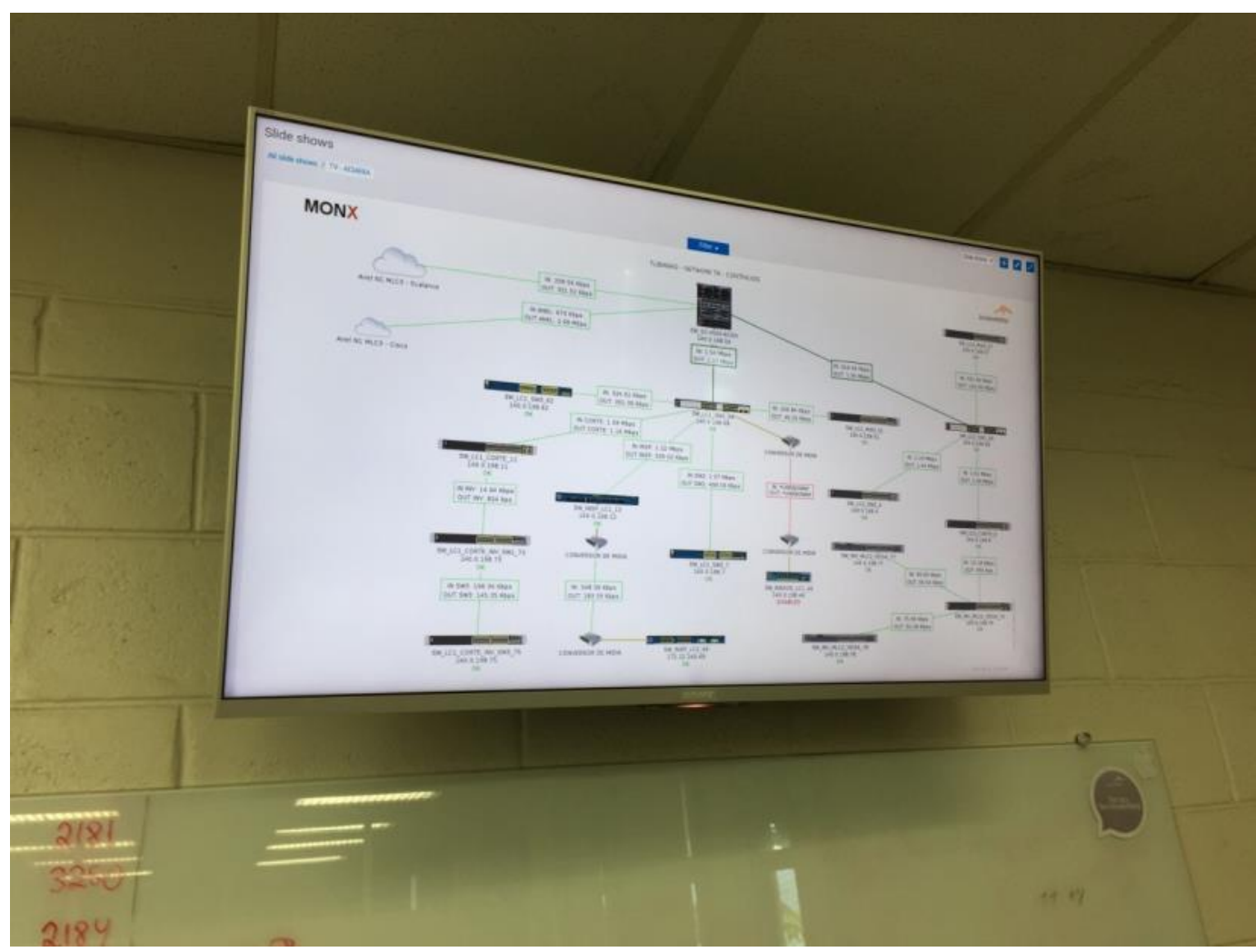

Figura 7. TV's com os Cockpits customizados

\subsection{2 - Ferramentas para complementação das análises}

Além das ferramentas de gerencia e coleta de informações, é feito o uso de outras ferramentas como o Grafana ${ }^{4}$ para confecção de dashboards mais elaborados:

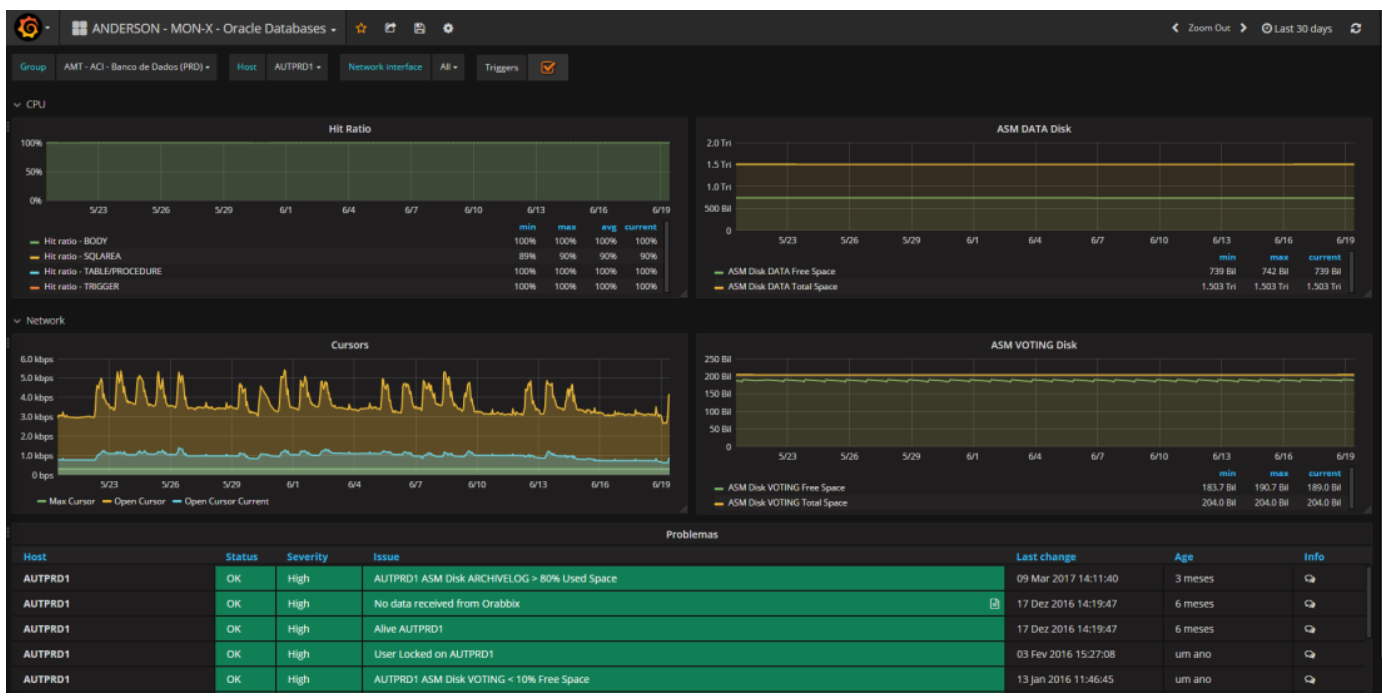

Figura 8. Interface do Grafana integrada ao Sistema de Monitoramento 


\subsection{Gestão de Alarmes}

O sistema pode enviar mensagem para qualquer plataforma (Whatsapp, Telegram, e-mail, SMS, entre outras) ou desencadear qualquer tipo de evento (criação automática de incidente no helpdesk, ação automática em algum ativo/sistema, etc...)

Além dessa grande flexibilidade no envio de alarmes, ele ainda possui toda uma gestão de alarmes que permite com que vários operadores de localidades distintas, trabalhem na mesma ferramenta com total integração. Permitindo não só o controle dos alarmes, como também visualização de histórico e comentários para cada evento.

\subsection{Atuação Automática em Sistemas e Dispositivos}

Com o mapeamento de alguns processos críticos, o próprio sistema já responde automaticamente para ações pré-determinadas.

Uma falha em um sistema ou dispositivo pode gerar uma reação do sistema que faz a correção automática sem a necessidade de intervenção do especialista.

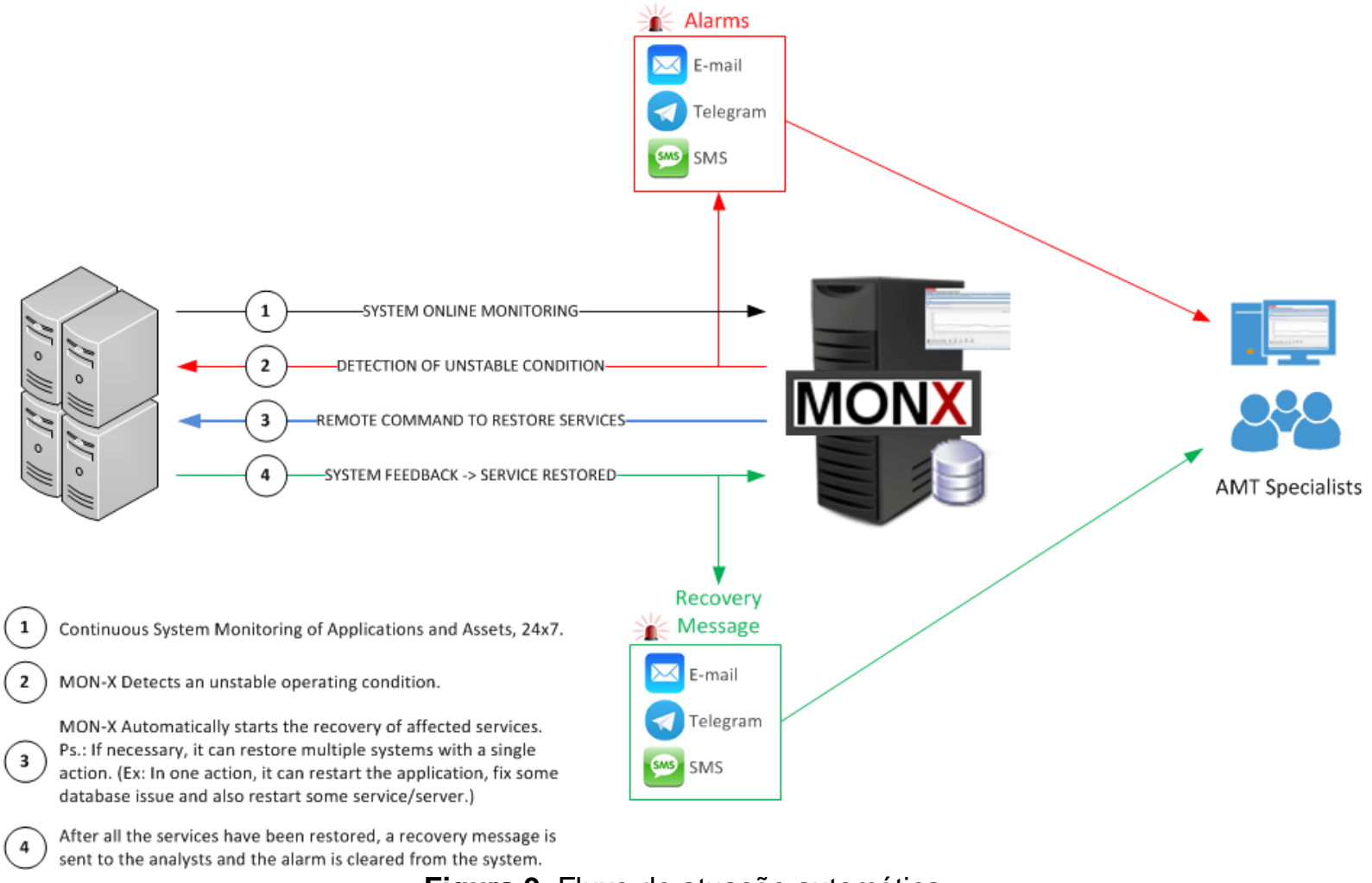

Figura 9. Fluxo de atuação automática

\subsection{Análise Preditiva}

Embora ainda esteja em fase de implementação de funções analíticas preditivas, a ferramenta a partir da versão 3.0 fornece uma grande gama de funções para análise preditiva. 


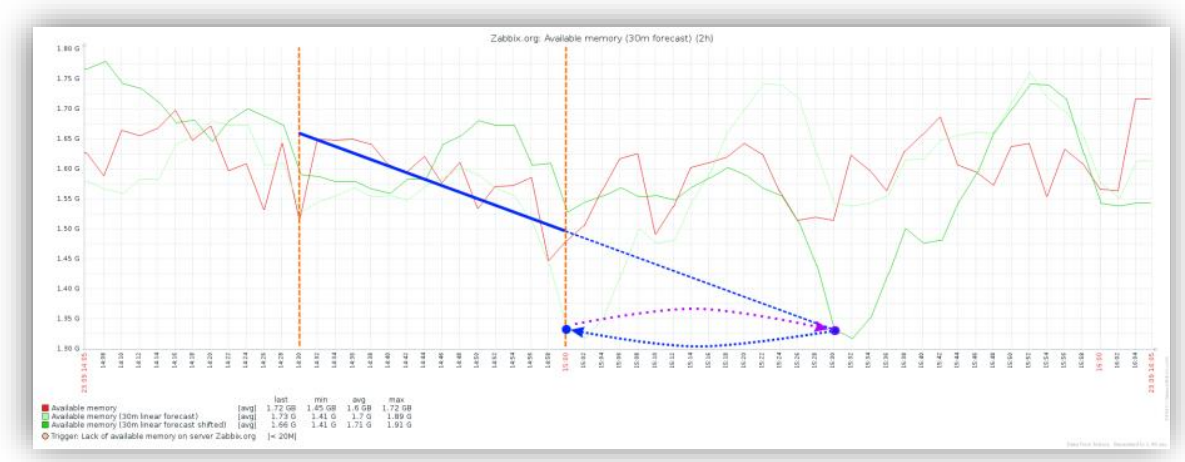

Figura 10. Exemplo de um trigger preditivo no Zabbix

\section{RESULTADOS E DISCUSSÃO}

\subsection{Resultados}

A ferramenta apresentou resultados em todas as áreas implantadas e foi selecionado o obtido no Lingotamento Contínuo 3 como exemplo para a apresentação. O caso abaixo envolve monitoramento de hardware legado (Alpha Server), sistema operacional legado (OpenVMS), Banco de Dados Oracle e o processo de Lingotamento Contínuo:

\section{Lingotamento Contínuo 3}

\section{ArcelorMittal}

Problema do Lingotamento Contínuo Máquina 3 (VMS / Oracle)

Problema: Problema de Travamento da Máquina do Contínuo 3 (desde seu start-up) O que o Zabbix Contribuiu: Ajudou a identificar as causas raízes e a lógica de quando ocorria o problema possibilitando a atuação e correção no código da aplicação.

\section{ANTES}

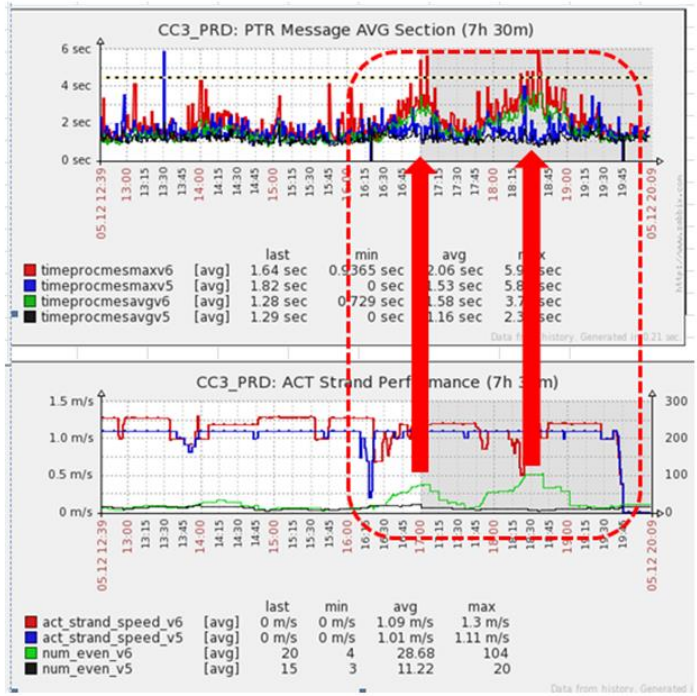

DEPOIS

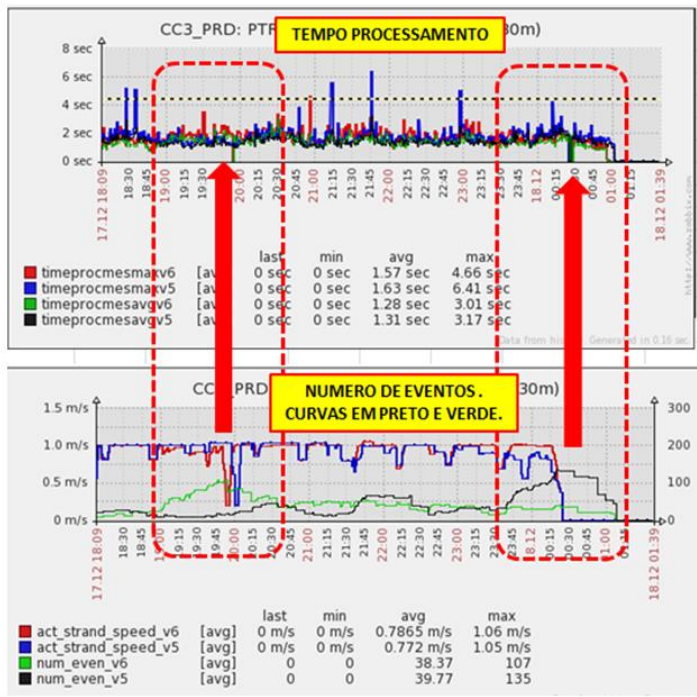

Figura 11. Resultado de um troubleshoot executado com apoio do MON-X 


\section{Benefícios da Solução de Monitoramento das Aplicações da Automação de Processos - Case Lingotamento Continuo 3}

ArcelorMittal

Tempo Parada e Atraso de Máquina

Minutos

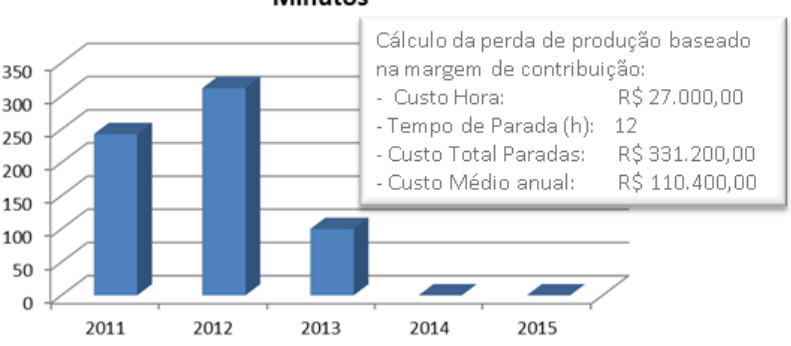

Tempo de Atendimento dos Incidentes Minutos

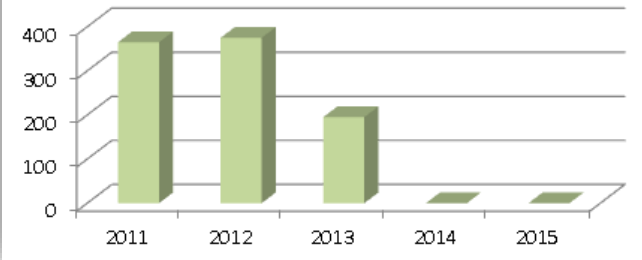

Quantidade de Incidentes

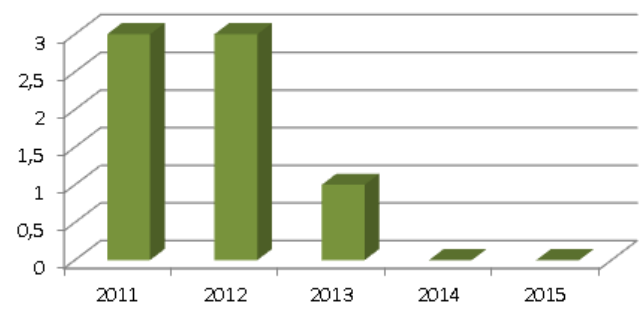

Aumento de Produtividade da Equipe Melhorias Implantadas

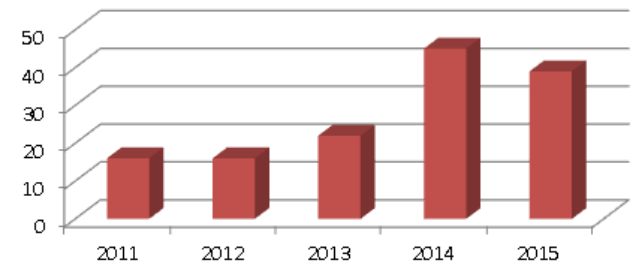

Figura 12. Resultado Financeiro de um troubleshoot executado com apoio do MON-X

\section{CONCLUSÃO}

Com a escolha de produtos extremamente customizáveis, foi possível em um curto espaço de tempo, construir uma solução abrangente e que cobre grande parte das demandas de monitoramento das áreas.

Esta solução tem o foco em dados estruturados e com bases de dados relacionais. Tendo isso em mente, fica subentendido que seu foco é em monitoramento de dados com baixa frequência de coleta. ( $>=30 \mathrm{~s}-1 \mathrm{~m}$ ). Para dados com alta frequência de coleta $(<30$ s), ainda são utilizadas bases temporais como o PIMS e quando seus dados são necessários, é feita a extração através de JDBC para o MON-X.

\section{REFERÊNCIAS}

1 Zabbix,

Zabbix

Documentation

Disponível

em: <https://www.zabbix.com/documentation/3.0/manual>. Acesso em 19/06/2017

Hewlett Packard, OpenVMS

Documentation

Disponível

em: <http://h41379.www4.hpe.com/doc/os84_index.html>. Acesso em 19/06/2017 Hewlett Packard, Tru64 Documentation

Disponível em: <http://h41361.www4.hpe.com/docs/pub_page/doc_list.html>. Acesso em 19/06/2017

4 Grafana Labs, Frafana Documentation - Disponível em: <http://docs.grafana.org/>. Acesso em 19/06/2017 